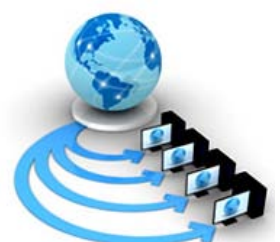

Volume 9, No. 2, March-April 2018

International Journal of Advanced Research in Computer Science

REVIEW ARTICLE

\author{
Available Online at www.ijarcs.info
}

\title{
COMPREHENSIVE STUDY OF APPLICATIONS OF SPATIO TEMPORAL DATA MINING IN GIS
}

\author{
Rajat Malik \\ Ph.D. Scholar \\ Department of Computer Science and Engineering \\ UIET, MDU, Rohtak, India
}

\author{
RainuNandal \\ Assistant professor \\ Department of Computer Science and Engineering \\ UIET, MDU, Rohtak, India
}

\begin{abstract}
The advancement in database and internet technology has led to large debris of data being collected. The process of extraction knowledge led to coining of new term called data mining. In this paper we have discussed the spatio temporal dimension of data mining and its applications in field of Geographical Information Systems. The applications include forest fires, Maize yield, Weather forecasting etc.
\end{abstract}

Keywords: Spatio, Temporal, Data, Mining, Geographical, Information, Systems

\section{INTRODUCTION}

The advancement in technology has led to large amount of data being accumulated, not all the data is useful. The process of retrieval of useful knowledge from the data is known as Data Mining. There are various parameters linked with the data like spatial parameters including location and temporal parameters including time at which measurement was made. [12][14]The improvement in sensor technology also had large impact on the field of Spatio temporal Data mining as data from these networks contain both these parameters. The ST data has various applications ranging from the field of Climate Science, Traffic to environment. The basic steps of data mining include

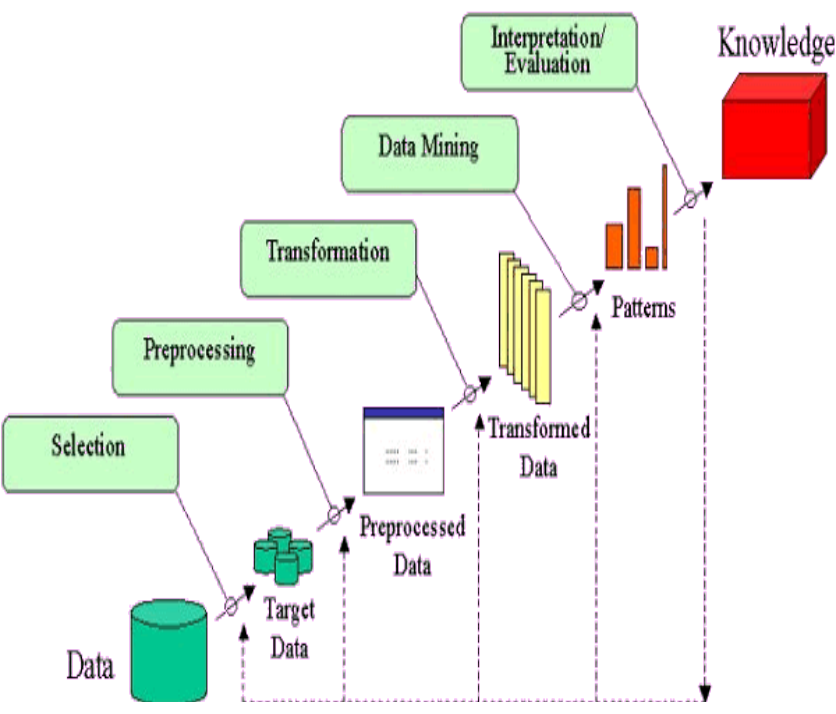

Figure 1knowledge Discovery Process [11]

The terms Data mining and KDD are used in place of one another but in real sense Data Mining is the single step in KDD process [11].

KDD is the overall process of getting knowledge from the data.
Data Mining is the process of getting patterns from the data.

\section{SPATIO TEMPORAL DATAMINING}

The data can have multiple dimensions like space and time. The large amount of this spatio temporal data come from search engine,GPS etc. The task of spatio temporal datamining can be shown as

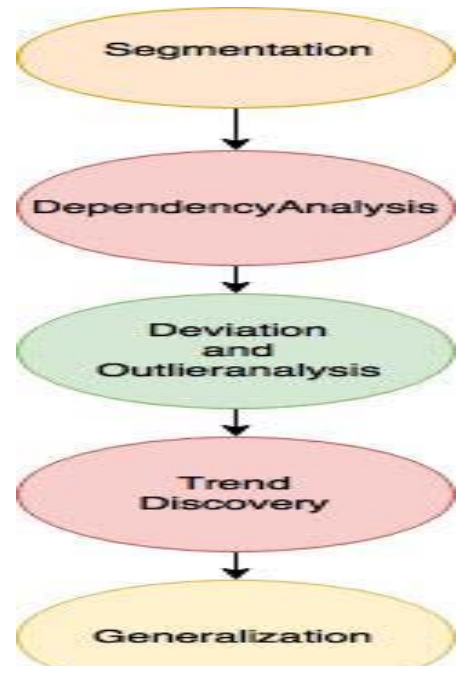

Figure 2Spatio Temporal Datamining Process[12]

\section{APPLICATION OF SPATIO TEMPORAL DATA MINING}

1. Climate Science: The changes in climate can affect the lives drastically, Studying the patterns and predicting the changes accordingly can help serve the mankind a better way and STDM is one way to it. [7]

2. Agriculture: The STDM also helps in field of agriculture by helping in improving the yield and detecting plant diseases. [2]

3. Neuroscience: The brain data has both the parameters of space and time. The technologies like MRI, EEG are used for collecting data. The analysis of these help in 
understanding the brain in better way and helps in finding the causes of disruptions.

4. Traffic Analysis: High traffic is causing lot of trouble; the analysis of these data can help administration to take necessary steps.

5. Market Analysis: The Data from companies if analyzed properly helps in understanding customer behavior and also helps in predicting the needs of customer.

6. Crime Data: The analysis of the previous crime data can help in reducing the crime rate and helping the Law Enforcement Agencies.

\section{SPATIO TEMPORAL DATA MINING IN GIS}

The geographical data can solve many problems of present day if analyzed properly [13]. The problems like forest fire, typhoons can be dealt in better way if we develop proper methodology for them. The prediction of these beforehand can solve many a problem.

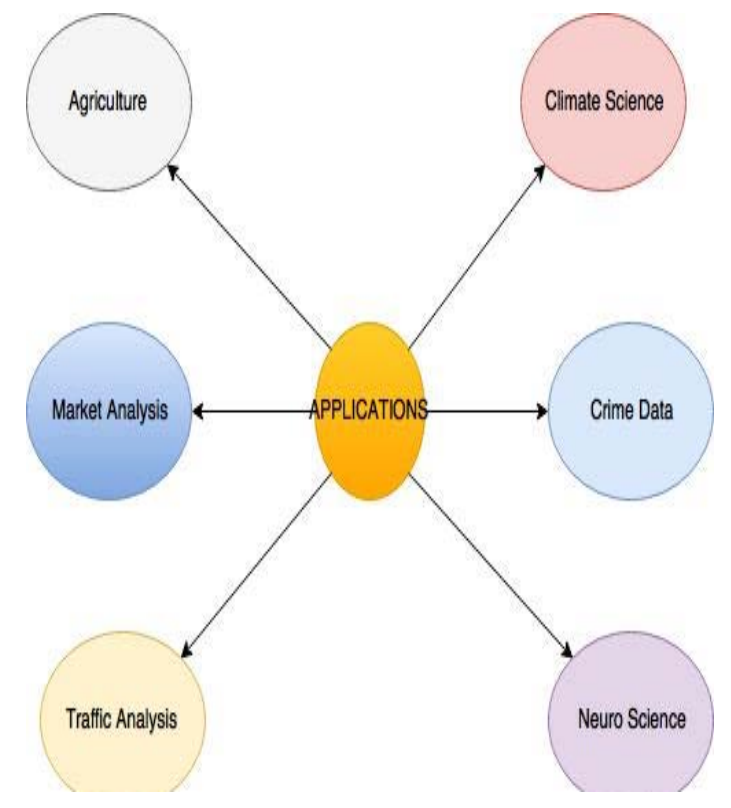

Figure 3Applicatios Of Spatio Temporal Data[10]

\section{LITERATURE SURVEY}

Tao Cheng and Jiaqiu Wang have discussed the important issue of forest fire and devised a framework for prediction of forest fire known as Integrated spatio-temporal forecasting framework. It uses a neural network which is dynamic and recurrent in nature. The forecasting is based on historical observations. The results of this protocol is better than the other protocols that is they have higher accuracy for shortterm prediction. This scheme has handled only one variable and in many cases, we may have different variables. This methodology can be used for other application domains [1].

Liying Cao et al. have discussed an approach for prediction of maize yield using spatio temporal data mining. This method does two things first one being the prediction of the sequence of target objects

and other one being the calculation of the effect of spatial distribution of soil-fertility on yield. The method uses the concept of neural network and linear regression. The error rate in the method is about $5 \%$. This is one of the fewest method that considers both time and space constraints. And results showed the significant increase in production [2].

AsanobuKitamoto has discussed about the approaches for collection of data for Typhoons

from satellites using spatio -temporal data mining. The principal component analysis is used for the spatial mining. The results revealed that the clustering procedures gave" birds-eye-view" visualisation. Also, a basic method for prediction of typhoons, that is Image mining Environment for typhoon analysis and prediction(IMET) is proposed, It uses K-NN and Euclidian metric to search for patterns [3].

BalakrishnaGokarajuet al. have discussed a scheme for detection of Harmful Algal Blooms,

These blooms pose threat to habitation. The proposed scheme is based on Machine Learning, The SVM is used a classifier. Through the data sets the prediction was efficient and the false alarms were very less [4].

$\mathrm{Xi}$-yong HOU et al. have discussed the issue of vegetation degradation. The circum-Bohai-Sea region is taken as the case study. The methodology used is Unary Linear Regression Model and Hust index. The results showed that the degraded area is mostly distributed in metros, coastalzone [5].

Sheng-Tun Li et al. have discussed about the cluster analysis in evaluation of air pollution.The SOM neural networks and multi-scale Wavelet transforms are used for this purpose. The key point to be noted here is that the scale plays an important role in cluster analysis. The result showed that due to increase in population and industrialization the places have more pollutants [6].

FolorunshoOlaiya and Adesesan Barnabas Adeyemo have discussed how data mining can be used for weather prediction. Artificial neural network and Decision tree algorithms are used.The classifier was trained using historical data to make predictions. The actual data and results are compared. The result was, if enough data is given to ANN to learn the prediction can be done in efficient way [7].

Fenzhen $\mathrm{Su}$ et al have discussed the impact of environmental factorson distribution of fishes. The model used here is STAMM(Spatio Temporal assignment mining model). Themethodology uses neighbourhood rule These along with relationships are used to create decision tables.The temperature is used as a parameter[8]

UlanbekTurdukulov et al. have used the pattern generated moving flocks as frequent pattern. Their comparison with other real data sets, the results were far better than previous techniques [9].

\section{CONCLUSION AND FUTURE SCOPE}

The spatio temporal dimensions of data if dealt properly can help serve mankind in better way. We have analysed that the Artificial Intelligence techniques including Machine 
Learning, Neural Networks etc. play a major role in this. This field has vast scope in predicting the events from the GIS data in more optimal way.

\section{REFERENCES}

[1]. Cheng, T. and Wang, J., 2008. Integrated Spatio-temporal Data Mining for Forest Fire Prediction. Transactions in GIS, 12(5), pp.591-611.

[2]. Cao, L., San, X., Zhao, Y. and Chen, G., 2013. The application of the spatio-temporal data mining algorithm in maize yield prediction. Mathematical and Computer Modelling, 58(3-4), pp.507-513.

[3]. Kitamoto, A., 2002. Spatio-temporal data mining for typhoon image collection. Journal of Intelligent Information Systems, 19(1), pp.25-41.

[4]. Gokaraju, B., Durbha, S.S., King, R.L. and Younan, N.H., 2011. A machine learning based spatio-temporal data mining approach for detection of harmful algal blooms in the Gulf of Mexico. IEEE Journal of selected topics in applied earth observations and remote sensing, 4(3), pp.710-720.

[5]. Hou, X.Y., Han, L., Gao, M., Bi, X.L. and Zhu, M.M., 2010, August. Application of spatio-temporal data mining and knowledge discovery for detection of vegetation degradation. In Fuzzy Systems and Knowledge Discovery (FSKD), 2010 Seventh International Conference on (Vol. 5, pp. 2124-2128). IEEE.

[6]. Li, S.T., Chou, S.W. and Pan, J.J., 2000, January. Multiresolution spatio-temporal data mining for the study of air pollutant regionalization. In System Sciences, 2000.
Proceedings of the 33rd Annual Hawaii International Conference on (pp. 7-pp). IEEE.

[7]. Olaiya, F. and Adeyemo, A.B., 2012. Application of data mining techniques in weather prediction and climate change studies. International Journal of Information Engineering and Electronic Business, 4(1), p.51.

[8]. Su, F., Zhou, C., Lyne, V., Du, Y. and Shi, W., 2004. A data-mining approach to determine the spatio-temporal relationship between environmental factors and fish distribution. Ecological Modelling, 174(4), pp.421-431.

[9]. Turdukulov, U., Calderon Romero, A.O., Huisman, O. and Retsios, V., 2014. Visual mining of moving flock patterns in large spatio-temporal data sets using a frequent pattern approach. International Journal of Geographical Information Science, 28(10), pp.20132029.

[10].Atluri, G., MacDonald III, A., Lim, K.O. and Kumar, V., 2016. The Brain-Network Paradigm: Using Functional Imaging Data to Study How the Brain Works. computer, 49(10), pp.65-71.

[11].http://www2.cs.uregina.ca/ dbd/cs831/notes/kdd/1_kdd. html

[12].Yao, X., 2003, November. Research issues in spatiotemporal data mining. In Workshop on Geospatial Visualization and Knowledge Discovery, University Consortium for Geographic Information Science, Virginia (pp. 1-6).

[13].https://researchguides.library.wisc.edu/c.php?g=178144 $\& p=1169699$

[14].Andrienko, G., Malerba, D., May, M. and Teisseire, M., 2006. Mining spatio-temporal data. 